\section{Indolent EBV-positive T-cell lymphoproliferative disorder arising in a chronic pericardial hematoma: the T-cell counterpart of fibrin-associated diffuse large B-cell lymphoma?}

Epstein-Barr virus-positive (EBV+) lymphoproliferative disorders (LPD) encompass a wide spectrum of disease entities ranging from indolent, self-limiting, and localized conditions to highly aggressive lymphomas. ${ }^{1,2}$ Fibrinassociated diffuse large $\mathrm{B}$-cell lymphoma (FA-DLBCL) is a recently recognized $\mathrm{EBV}+\mathrm{B}$-cell lymphoma associated with a presumably restricted immune-privileged space..$^{3-6}$ It is unknown whether T lymphocytes can also proliferate and be transformed in a similar setting. In this study, we reported a unique case of indolent EBV+ T-cell LPD arising in a chronic pericardial hematoma and sharing many clinicopathological features with FA-DLBCL. This case may help expand the spectrum of EBV+ LPD associated with a local immunodeficiency.

A 64-year-old man complained of chest tightness and shortness of breath. A computed tomography scan showed an isolated $8.6 \times 3.7 \mathrm{~cm}$ pericardial mass without lymphadenopathy or any additional lesions. Surgical resection revealed an organized pericardial hematoma closely adherent to the right atrium and right ventricle in the anterior mediastinum. A further inquiry revealed an 18-year history of pericardial hematoma, for which the first surgical evacuation was performed 2 year earlier in an outside hospital with a pathology diagnosis of chronic hematoma. However, the patient had a pericardial relapse 1 year later. In addition, the patient had a left lower lobectomy for a pulmonary abscess about 30 years ago, and myocarditis about 40 years ago. After the current complete excision, the patient opted for a watchand-wait approach. He was well without any clinical and radiologic evidence of recurrence or progression of the disease in the next 18 months of follow-up.

Gross examination of the current specimen revealed a hematoma with a wall up to $0.4 \mathrm{~cm}$ of thickness, and old blood as well as a blood clot adherent to its luminal side, measuring $9.0 \times 7.8 \times 4.0 \mathrm{~cm}$ in total. No gross tumor was identified. Microscopic examination revealed a fibrous wall and a content of fibrinous and hemorrhagic material. Foci of dystrophic calcification and patchy infiltrates of chronic inflammatory cells were seen in the outer layer of the fibrous wall. In the 6 of 30 sampled tissue blocks, multiple small clusters of atypical lymphoid cells were found embedded within the fibrin deposition or lining up within the inner layer of the fibrous wall, with each cluster measuring no more than $2.0 \mathrm{~mm}$. The atypical cells were medium-sized and hyperchromatic with round or irregular nuclei and moderate amount of cytoplasm, some of which appeared degenerated (Figure 1A-C).
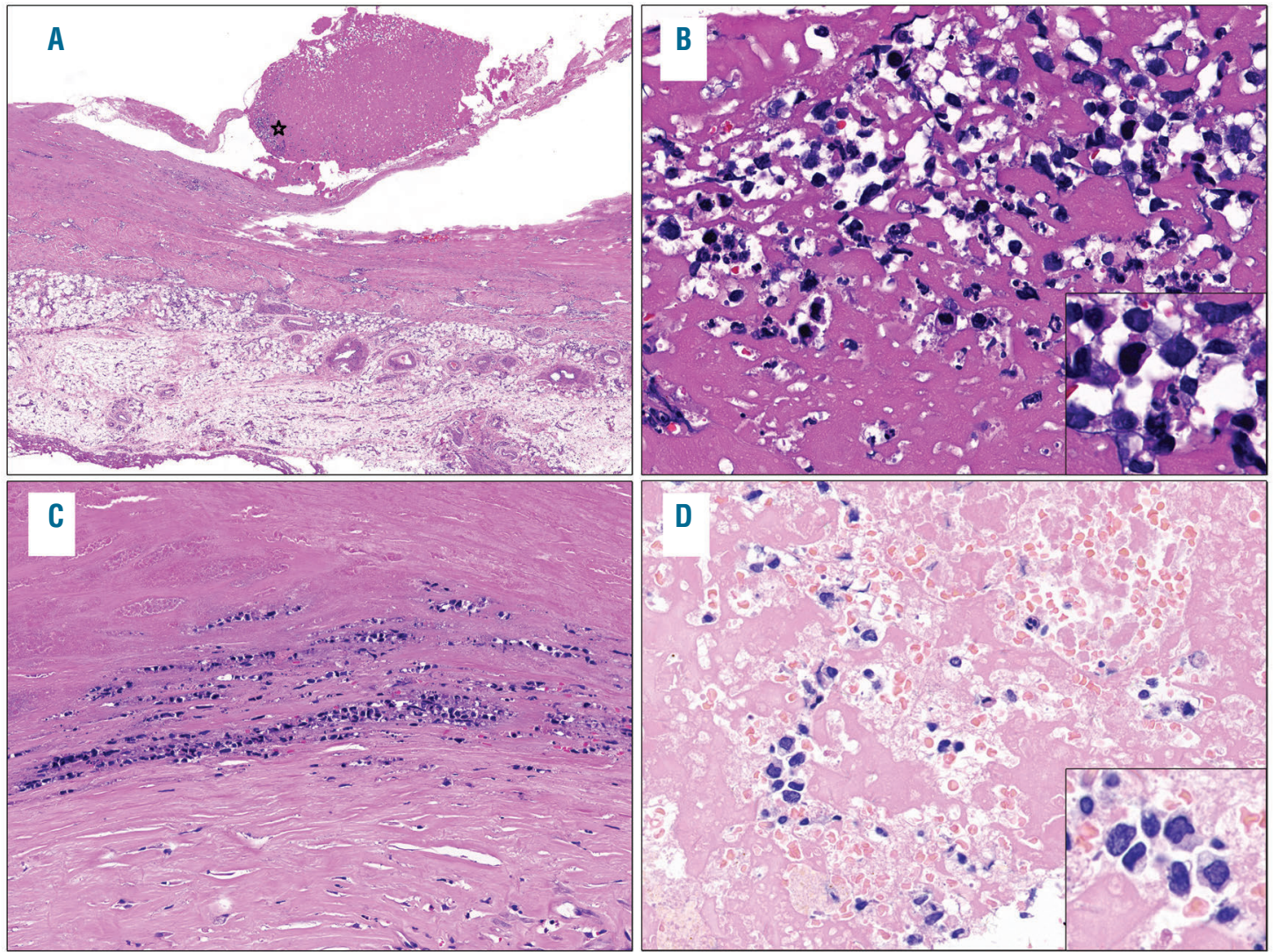

Figure 1. Epstein-Barr virus-positive T-cell lymphoproliferative disorders arising in chronic pericardial hematoma. (A-C) The current specimen. (A) Low power view of luminal fibrinous content with a microscopic focus of atypical lymphoid cells (indicated by *) and fibrous wall. (B) The atypical lymphoid cells show round or irregular nuclei and moderate amount of cytoplasm, some of which appear degenerated. The insert shows cytological details of the atypical cells. (C) The atypical cells line up within the inner fibrous wall. (D) The small to medium-sized atypical lymphoid cells within the acellular liquid in the prior specimen. The insert shows cytological details of the atypical cells. Original magnification: (A) 20X. (C) 200X. (B, D) 400X. 


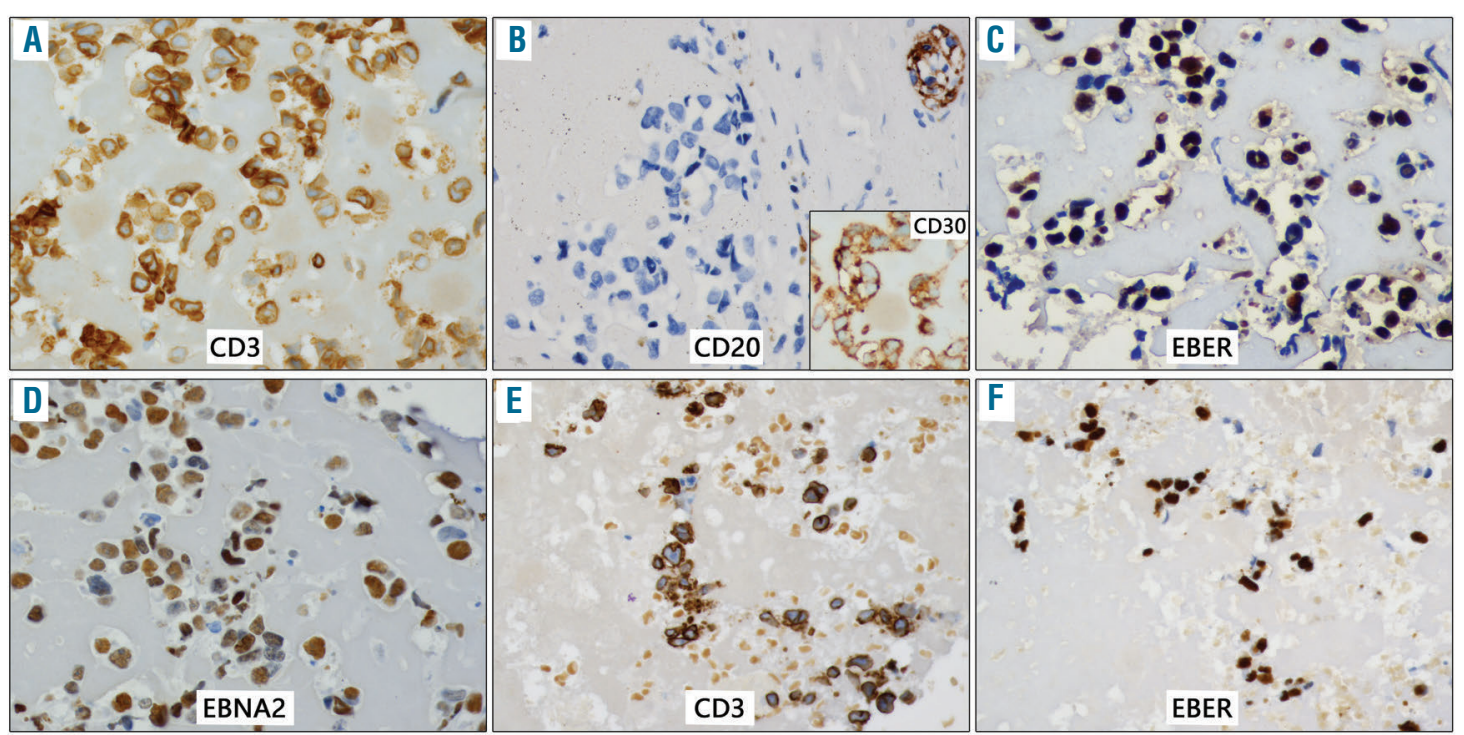

Figure 2. Immunohistochemical analyses and in situ hybridization for Epstein-Barr virus-encoded small RNA (EBER). (A-D) The atypical lymphoid cells in the current specimen are positive for CD3 (A), CD30 (B, insert), EBER (C), and EBNA2 (D), and negative for CD20 (B). (E, F) The atypical lymphoid cells in the prior specimen are positive for CD3 $(E)$ and EBER $(F)$. Original magnification: (A-F) 400X.

Microscopic examination of the prior specimen revealed few scattered small to medium-sized hyperchromatic lymphoid cells in the acellular fluid in the 1 of 3 sampled blocks (Figure 1D).

Immunohistochemical analyses as well as in situ hybridization for EBV-encoded small RNA (EBER) were performed on the current and prior specimens with similar findings. The atypical lymphoid cells were positive for CD3, CD30, CD43, TIA-1, MUM1, BCL-2, EBNA2, and EBER ( $>90 \%$ of CD3+ cells), and were negative for CD20, CD79a, PAX-5, OCT-2, BOB-1, CD2, CD4, CD5, CD7, CD8, CD10, CD38, CD56, granzyme B, perforin, BCL-6, PD-1, ICOS, ALK, EMA, HHV-8, and LMP-1 (Figure 2). B-cells were rare. The Ki-67 proliferation index was $>90 \%$. PCR analysis performed on the current specimen using the primer system of BIOMED-2 standard showed a monoclonal TCR- $\gamma$ chain gene rearrangement. However, the number of atypical cells in the prior specimen was too low for TCR rearrangement analysis.

EBV infection is the most common persistent viral infection in humans affecting approximately $95 \%$ of the world's population. Most of the infected people have an asymptomatic life-long carrier status. This sustained latent infection results from a finely regulated balance between the biologic properties of EBV and the host immune status, and the disruption of this balance can give rise to a variety of EBV+ $\mathrm{LPD}^{7}$ FA-DLBCL is an unusual type of EBV+ large B-cell lymphoma, which is not mass-forming and thus does not directly produce symptoms, and is discovered incidentally on histologic examination of surgical specimens excised for various pathologies other than lymphoma. ${ }^{8}$ It is incorporated into 2017 World Health Organisation classification as a subtype of DLBCL associated with chronic inflammation. However, recent studies have suggested that FA-DLBCL may be recognized as a form of EBV+ LPD with an alternative pathogenesis rather than a lymphoma. It may be associated with a restricted immune-privileged space where EBV-infected B cells are protected from the cyto- toxic effects of EBV-specific T cells and allowed to proliferate unchecked. Nevertheless, the transformed B cells may lack the ability to grow autonomously outside. ${ }^{1,2,5,6}$

The EBV+ T-cell LPD we reported here arose in a chronic pericardial hematoma. Similarly, FA-DLBCL are often seen arising in chronic hematomas or in other similar settings, such as cardiac myxoma, fibrin thrombus, and pseudocysts. ${ }^{4,6,-11}$ Pathologically, the atypical lymphoid cells in our case were incidentally identified in small aggregates within the fibrinous material with an increase in number and size in the current specimen but without evidence of spread beyond the hematoma. The localized nature and the lack of relapse or progression after the second excision indicate an indolent clinical course. Moreover, these cells showed definite atypia, an aberrant immunophenotype (loss of multiple T-cell markers CD2, CD4, CD5, CD7, and CD8), a high proliferation rate, a diffuse positivity of EBER and EBNA2, and a monoclonal antigen receptor gene rearrangement. All these findings in this case were reminiscent of FA-DLBCL. It should be noted that the EBV latency profile (LMP1/EBNA2+) in this case was unusual. However, this latency profile has been reported in cases of FA-DLBCL. ${ }^{3-6}$ In addition, latency profiles intermediate between latencies I and II have also been described. ${ }^{12}$

In the light of the clinicopathological similarities between this case and FA-DLBCL, we believe that there may be some commonalities in their pathogenesis. If the restricted immune-privileged space does exist and can give rise to FA-DLBCL, it is reasonable to expect that $\mathrm{EBV}+\mathrm{T}$-cell LPD can arise in a similar clinical setting by escaping the cytolytic response of host to EBV-infected T cells and allowing their proliferation and transformation. Studies of the pathogenesis of FA-DLBCL shed light on the pathogenesis of this case. We believe that this case may represent the T-cell counterpart of FA-DLBCL. By analogy, breast implant-associated anaplastic large cell lymphoma (ALCL), another type of T-cell lymphoma that develops in a limited space, shares some clinicopatholog- 
ical features with FA-DLBCL and this case including an association with local chronic inflammation, locally altered immune state, and an indolent clinical course as in most cases, although EBV+ cases, has never been found so far. ${ }^{13}$ Interestingly, cases of breast implant-associated EBV+ B-cell lymphoma have also been reported recently, which are morphologically similar to breast implant-associated ALCL or lymphomatoid granulomatosis. ${ }^{14}$ Menter et al. recently reported a case of ALKnegative ALCL with a STAT6 mutation arising in the recurrent thrombi and preceded by clonally related lymphomatoid papulosis. This case demonstrated many common clinicopathological features with breast implant-associated ALCL, breast implant-associated $\mathrm{EBV}+\mathrm{B}$-cell lymphoma, and the EBV+ T-cell LPD we reported here. It was speculated that the circulating lymphomatoid papulosis cells may be transformed into an overt lymphoma in an immunoprivileged niche in the thrombotic material. ${ }^{15}$ In summary, multiple lines of evidence support that both T- and B-cell LPD can develop in similar clinicopathological settings, which supports our hypothesis in our case report.

The case we reported here did not fit well into any of these well-defined categories of EBV+ LPD in the current World Health Organisation classification. However, it is important to distinguish this case from these EBV+ entities, especially extranodal NK/T-cell lymphoma, nasal type, and EBV+ B-cell lymphoma. Extranodal NK/T-cell lymphoma often involves the upper aerodigestive tract, skin, gastrointestinal tract, and testes, and is characterized by vascular damage and destruction, prominent necrosis, and a cytotoxic phenotype. ${ }^{8}$ Obviously, this case can be easily distinguished from extranodal NK/T-cell lymphoma based on its distinctive clinicopathological features. Rare cases of EBV+ B-cell lymphoma with aberrant expression of $\mathrm{T}$-cell markers and/or cytotoxic molecules should also be taken into account in the differential diagnosis. ${ }^{3,13}$ Nevertheless, the T-cell origin of this case was confirmed by its immunophenotype as well as the Tcell receptor rearrangement.

In summary, we describe the first case of indolent EBV+ T-cell LPD arising in a chronic pericardial hematoma and sharing many clinicopathological features with FA-DLBCL. It is reasonable to hypothesize that this case may represent the T-cell counterpart of FA-DLBCL. Importantly, with the report of this case, pathologists will be alerted to this diagnostic possibility and similar cases may follow in the future.

Zhen Wang," Qixing Gong, 1," Youcai Zhao, ${ }^{2}$ Hai Xu, Shimin $\mathrm{Hu}^{4}$ and Zhihong Zhang

${ }^{1}$ Department of Pathology, Jiangsu Province Hospital and the First Affiliated Hospital of Nanjing Medical University, Nanjing, China; 'Department of Pathology, Nanjing First Hospital, Nanjing, China; ${ }^{3}$ Department of Radiology, Jiangsu Province Hospital and the First Affiliated Hospital of Nanjing Medical University, Nanjing, China and ${ }^{4}$ Department of Hematopathology, the University of Texas MD Anderson Cancer Center, Houston, TX, USA

'ZW and QG contributed equally as co-first authors.
Correspondence: ZHIHONG ZHANG

zhangzh@njmu.edu.cn

doi:10.3324/haematol.2020.252007

Information on authorship, contributions, and financial \& other disclosures was provided by the authors and is available with the online version of this article at www. haematologica.org.

\section{References}

1. Dojcinov SD, Fend F, Quintanilla-Martinez L. EBV-positive lymphoproliferations of $\mathrm{B}$ - $\mathrm{T}$ - and NK-cell derivation in non-immunocompromised hosts. Pathogens. 2018;7(1):28.

2. Rezk SA, Zhao X, Weiss LM. Epstein-Barr virus (EBV)-associated lymphoid proliferations, a 2018 update. Hum Pathol. 2018;79:18-41.

3. Loong F, Chan AC, Ho BC, et al. Diffuse large B-cell lymphoma associated with chronic inflammation as an incidental finding and new clinical scenarios. Mod Pathol. 2010;23(4):493-501.

4. Gruver AM, Huba MA, Dogan A, et al. Fibrin-associated large B-cell lymphoma: part of the spectrum of cardiac lymphomas. Am J Surg Pathol. 2012;36(10):1527-1537.

5. Boroumand N, Ly TL, Sonstein J, et al. Microscopic diffuse large Bcell lymphoma (DLBCL) occurring in pseudocysts: do these tumors belong to the category of DLBCL associated with chronic inflammation? Am J Surg Pathol. 2012;36(7):1074-1080.

6. Boyer DF, McKelvie PA, de Leval L, et al. Fibrin-associated EBV-positive large B-cell lymphoma: an indolent neoplasm with features Ddstinct from diffuse large B-cell lymphoma associated with chronic inflammation. Am J Surg Pathol. 2017;41(3):299-312.

7. Taylor GS, Long HM, Brooks JM, et al. The immunology of EpsteinBarr virus-induced disease. Annu Rev Immunol. 2015;33:787-821.

8. Swerdlow SH, Campo E, Harris NL, et al. WHO Classification of Tumors of Hematopoietic and Lymphoid Tissues, Revised 4th ed. Lyon: IARC. 2017.

9. Sugita Y, Ohta M, Ohshima K, et al. Epstein-Barr virus-positive lymphoproliferative disorder associated with old organized chronic subdural hematoma. Pathol Int. 2012;62(6):412-417.

10. Hayes C, Alkan S, Kitahara S. Indolent fibrin-associated EBV-positive large B cell lymphoproliferative disorder in a lower extremity aneurysmal hematoma: a case report. J Hematop. 2014;7(3):139-143.

11. Kameda K, Shono T, Takagishi S, et al. Epstein-Barr virus-positive diffuse large B-cell primary central nervous system lymphoma associated with organized chronic subdural hematoma: a case report and review of the literature. Pathol Int. 2015(3);65:138-143.

12. Shannon-Lowe C, Rickinson A. The global landscape of EBV-associated tumors. Front Oncol. 2019;9:713.

13. Quesada AE, Medeiros LJ, Clemens MW, et al. Breast implant-associated anaplastic large cell lymphoma: a review. Mod Pathol. 2019;32(2):166-188.

14. Rodríguez-Pinilla SM, Sánchez-García FJ, Balagué $O$, et al. Breast implant-associated EBV-positive large B-cell lymphomas: report of three cases. Haematologica. 2019 Nov 21. [Epub ahead of print]

15. Menter T, Ballova V, Caspar C, et al. ALK-negative anaplastic large cell lymphoma arising in the thrombus of an aortic prosthesis preceeded by clonally related lymphomatoid papulosis. Virchows Arch. 2019;474(6):763-767. 